\title{
Seletrvidade de SÁlvia (Salvia splendens) AO HeRBicida OXYfluorfen Veiculado à Palha de ARRoz ${ }^{1}$
}

\author{
Selectivity of Salvia splendens to Oxyfluorfen Applied to Rice Straw
}

PIVETTA, K.F.L. ${ }^{2}$, ROSA, C.S. ${ }^{3}$, PITELLI, R.A. ${ }^{4}$ e COAN, R.M. ${ }^{5}$

\begin{abstract}
RESUMO - O manejo de plantas daninhas em canteiros de floríferas é um dos principais aspectos que interferem na manutenção dos jardins. Dessa forma, este trabalho teve como objetivo estudar a possibilidade de veiculação do oxyfluorfen à palha de arroz e a seletividade da sálvia (Salvia splendens), uma das principais floriferas produzidas e comercializadas no Brasil, ao herbicida. O delineamento experimental foi em blocos casualizados, no esquema fatorial $4 \times 3$, com quatro repetições. Os tratamentos foram quatro quantidades de palha de arroz $\left(0,4,6\right.$ e 8 t ha $\left.^{-1}\right)$ combinadas com três doses do herbicida oxyfluorfen $\left(0,1\right.$ e $\left.2 \mathrm{~L} \mathrm{ha}^{-1}\right)$. Observou-se que os tratamentos que levaram à veiculação do herbicida (nas duas doses testadas e nas três quantidades de palha) apresentaram controle de plantas daninhas sem que efeitos fitotóxicos severos fossem observados nas plantas de sálvia. A pulverização direta do herbicida sobre o solo e as plantas de sálvia não se mostrou viável. O tratamento que proporcionou controle satisfatório de plantas daninhas sem causar danos às plantas de sálvia e que, por isso, pode ser recomendado foi o que recebeu o herbicida na dose de $2 \mathrm{~L} \mathrm{ha}^{-1}$, veiculado a $4 \mathrm{th} \mathrm{h}^{-1}$ de palha de arroz.
\end{abstract}

Palavras-chave: cobertura morta, efeitos fitotóxicos, planta ornamental.

\begin{abstract}
Weed control is one of the main constrains to flower garden maintenance. This work aimed to study the possibility of translocating oxyfluorfen to rice straw and of selectivity of Salvia splendens, one of the main ornamental species produced and commercialized in Brazil, to the herbicide. A randomized block design was used in the factorial scheme $4 \times 3$, with four repetitions. The treatments consisted of four amounts of rice straw $\left(0,4,6\right.$ and 8 t ha $\left.{ }^{-1}\right)$ combined with three doses of oxyfluorfen $\left(0,1\right.$ and $\left.2 \mathrm{~L} \mathrm{ha}^{-1}\right)$. The results showed that the treatments that led to translocation of the herbicide to rice straw showed great weed control, without any high phytotoxicty

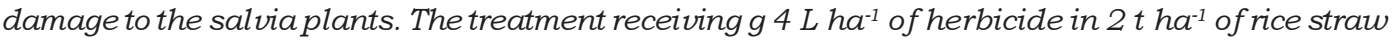
provided satisfactory results, without causing any phytotoxicity damage to the salvia plants and thus can be recommended for weed control.
\end{abstract}

Keywords: mulching, phytotoxicity effects, ornamental plant.

\section{INTRODUÇÃO}

A espécie Salvia splendens, também conhecida como sálvia ou alegria-dos-jardins, é uma planta nativa do Brasil, herbácea, perene, com flores vermelhas muito vistosas, notável para plantio em bordaduras e para formação de grandes maciços de plantas, sendo muito utilizada no paisagismo (Lorenzi \& Souza, 2001).

Em qualquer interferência paisagística, a manutenção, que deve ser periódica e qualificada, é uma das principais etapas, se não a principal. Nesse contexto, um dos aspectos

1 Recebido para publicação em 14.10.2007 e na forma revisada em 18.6.2008.

Parte da Dissertação de Mestrado da primeira autora.

${ }^{2}$ Enga-Agra ${ }^{2}$, Bromélias Rio, <camisrosa@yahoo.com.br>; ${ }^{3}$ Profa., Dra., UNESP/FCAV, Dep. de Produção Vegetal, Via de acesso Prof. Paulo Donato Castellane, s/n, 14884-900, Jaboticabal-SP, <kathia@fcav.unesp.br>; 4 Prof. Titular, UNESP/FCAV, <rapitelli@ecosafe.agr.br>; 5 Doutoranda, Programa de Produção Vegetal, UNESP/FCAV, <ruchelecoan@yahoo.com.br>. 
mais importantes é o manejo de plantas daninhas.

O controle manual de plantas daninhas ainda é a prática mais comum em jardins, porém é bastante onerosa, demorada e, na maioria das vezes, pouco eficiente, podendo ser dificultada pela localização e disposição dos canteiros. A alternativa mais viável é o controle químico, mas há poucas informações na literatura, principalmente relacionadas à seletividade de herbicidas às diferentes plantas ornamentais. Maciel et al. (2005) enfatizam a importância do controle químico de plantas daninhas em jardins, uma vez que proporciona rápidos resultados e economia na utilização de mão-de-obra.

Outro fator que contribui para a beleza do jardim, além do uso de espécies ornamentais adequadas e manutenção, é a utilização de materiais de origem vegetal ou mineral para a cobertura do solo, que complementam o visual, sendo, muitas vezes, até necessária em locais onde a cobertura vegetal não se desenvolve a contento; a palha de arroz é um dos materiais de origem vegetal que podem ser utilizados.

A cobertura morta também protege o solo das adversidades do clima (Creagur \& Katchur, 1975); regula o regime térmico do solo; conserva a água e o solo; reduz a perda de nutrientes por lixiviação (Carter \& Johnson, 1988), bem como a perda de água por evaporação e as oscilações da temperatura do solo (Bragagnolo \& Mielniczuk, 1990); melhora as qualidades fisicas e químicas do solo (Fialho et al., 1991); reduz a velocidade da enxurrada, promovendo menores perdas de água e solo (Alves et al., 1995); e contribui para o controle de plantas daninhas (Severino \& Christoffoleti, 2001).

Dependendo do material utilizado para cobertura morta, pode haver influência alelopática sobre a germinação de sementes de plantas daninhas. Araújo et al. (1993) estudaram o efeito alelopático de diferentes tipos de coberturas mortas na cultura do alho e verificaram que a utilização de palha de arroz promoveu redução no número de plantas daninhas. Semelhantemente, Santos et al. (2001) constataram que a palha de arroz proporcionou maior inibição na germinação de sementes de caruru (Amaranthus viridis) em lavoura de café.
Com o objetivo de unir os beneficios da utilização dos herbicidas e da cobertura morta, alguns autores têm estudado o controle de plantas daninhas por meio do uso de herbicidas associados a tipos de cobertura morta, em diferentes situações, entre eles Gravena et al. (2004) e Azania et al. (2004, 2006).

O herbicida oxyfluorfen é pouco solúvel em água e fortemente adsorvido nas partículas do solo, por isso é dificilmente lixiviado; fica na camada superficial e ali se decompõe, sem deixar resíduos perigosos, além de apresentar eficiente controle de várias espécies de plantas daninhas (Pereira, 1987). Yen et al. (2003) estudaram a dissipação e mobilidade do oxyfluorfen em diferentes solos sob diferentes condições de umidade e temperatura e concluíram que altas temperaturas aumentaram a taxa de dissipação; a possibilidade de contaminação de água subterrânea por oxyfluorfen é muito baixa e só é possivel quando o solo apresenta níveis muito baixos de matéria orgânica. Pelas suas características, vem sendo estudado no controle de plantas daninhas em ornamentais (Freitas et al., 2007a,b) sem que tenham sido observados efeitos fitotóxicos, quando a aplicação é dirigida.

Assim, o presente trabalho teve por objetivo verificar a veiculação do herbicida oxyfluorfen em palha de arroz e a sua seletividade à sálvia.

\section{MATERIAL E MÉTODOS}

O presente experimento foi instalado no Viveiro Experimental de Plantas Ornamentais e Florestais (Horto), pertencente à Faculdade de Ciências Agrárias e Veterinárias, UNESP/ FCAV, campus de Jaboticabal-SP, e conduzido no período de 7 novembro de 2006 a 8 de fevereiro de 2007.

O delineamento experimental utilizado foi o de blocos ao acaso com quatro repetições, em esquema fatorial $4 \times 3$. Os tratamentos foram constituídos pelas quantidades de palha de $\operatorname{arroz}\left(0,4,6\right.$ e $\left.8 \mathrm{t} \mathrm{ha}^{-1}\right)$ combinadas com as do herbicida oxyfluorfen $\left(0,1\right.$ e $\left.2 \mathrm{~L} \mathrm{ha}^{1}\right)$. Cada parcela tinha dimensões de $1 \mathrm{~m}^{2}$.

Foram erguidos quatro canteiros que representavam os blocos (repetições), acrescidos de $20 \%$ de composto orgânico preparado na 
UNESP/FCAV, de $14,0 \times 1,0 \mathrm{~m}$. Cada canteiro continha os 12 tratamentos de $1 \mathrm{~m}^{2} \mathrm{e}$, nas extremidades, bordaduras também de $1 \mathrm{~m}^{2}$.

Foi realizada análise química da mistura dos canteiros, no Laboratório de Análise de Solo e Planta, pertencente ao Departamento de Solos e Adubos da UNESP/FCAV. Devido às condições químicas favoráveis apontadas na análise do solo, não foi realizada adubação corretiva.

Para o ensaio, foram utilizadas mudas comerciais de sálvia com aproximadamente $30 \mathrm{~cm}$ de altura, adquiridas na empresa Prontaflora Garden Center, Cravinhos, SP. Realizou-se plantio manual 30 dias antes da introdução da cobertura morta + calda do herbicida nas parcelas experimentais, para o estabelecimento inicial das plantas no campo.

Cada parcela experimental foi constituída por quatro linhas de plantio, com cinco plantas cada, no espaçamento de $0,20 \mathrm{~m}$ entre as linhas, totalizando $1,0 \mathrm{~m}^{2}$; as linhas externas de cada lado e uma planta na extremidade de cada linha constituíram as bordaduras, resultando em seis plantas mensuráveis ou $0,08 \mathrm{~m}^{2}$ de área útil por parcela.

Uma comunidade infestante composta por Brachiaria decumbens (200 plantas $\left./ \mathrm{m}^{2}\right)$ e Amaranthus viridis (50 plantas $\mathrm{m}^{-2}$ ) foi semeada em cada parcela, a fim de garantir a presença de plantas daninhas.

Para os cálculos referentes à dosagem do herbicida veiculado na cobertura morta, determinou-se inicialmente o poder de embebição da casca de arroz, por meio da fórmula descrita em Moreira (1990):

$$
P E=[(P U-P S) \times 100] / P S
$$

em que:

$P E=$ poder de embebição da cobertura morta (\%);

$P S=$ peso da cobertura morta seca ao ar (kg); e

$P U=$ peso da cobertura morta embebida em água após o escorrimento da água gravitacional por 30 minutos.

O cálculo da concentração da fórmula comercial do herbicida na calda onde a palha de arroz foi embebida, para obtenção da dose de ingrediente ativo necessária, foi efetuado por meio da fórmula também descrita em Moreira (1990):

$$
C H F=\left(D I A \times 10^{10}\right) /(Q P \times P E \times C I A F)
$$

em que:

$C H F=$ concentração do produto comercial na calda de embebição da cobertura morta (ppm);

$D I A=$ dose de ingrediente ativo por hectare (kg ha-1);

$Q P=$ quantidade de cobertura morta (seca ao ar) a ser utilizada $\left(\mathrm{kg} \mathrm{ha}^{-1}\right)$; e

$C I A F=$ concentração do ingrediente ativo na fórmula comercial do herbicida (\%).

De acordo com experimentação prévia, a palha de arroz de cada parcela foi preparada separadamente, utilizando-se sacos de plásticos. A calda do herbicida foi adicionada à palha de arroz presente no saco de plástico, o qual foi, em seguida, fechado e agitado manualmente por um período de cinco minutos. $\mathrm{Na}$ seqüência, os sacos foram abertos até a secagem da palha, em sala escura e temperatura ambiente. Após um periodo de 48 horas, a palha estava seca e pronta para ser colocada nos tratamentos experimentais.

A aplicação do herbicida nos tratamentos que não receberam aplicação da palha de arroz foi realizada em pré-emergência das plantas daninhas, com auxílio de um pulverizador costal pressurizado a ar comprimido, na pressão de 30 lbf pol-2, acoplado a uma barra de dois bicos do tipo 11002 , proporcionando consumo de calda de $200 \mathrm{~L} \mathrm{ha}^{-1}$. A aplicação foi feita no período matutino, sem a presença de orvalho nas folhas. No momento da aplicação os termômetros registravam a temperatura do ar de $27,8{ }^{\circ} \mathrm{C}$ e umidade relativa do ar de $74 \%$, com ausência de ventos e nebulosidade em torno de $95 \%$. Uma proteção mecânica foi usada entre os tratamentos apenas para impedir qualquer deriva do produto. As mudas de sálvia se encontravam com $25-30 \mathrm{~cm}$ de altura, iniciando o florescimento.

Os efeitos fitotóxicos dos herbicidas foram determinados por meio de avaliações visuais das plantas de sálvia, aos 7, 14, 21, 28, 35, 42 e 56 dias após a aplicação (DAA), utilizando-se a escala desenvolvida pela EWRC (1964), com notas variando de 1 a 9 , em que 1 implica 
ausência de quaisquer injúrias e 9, morte das plantas. A altura das plantas de sálvia foi obtida utilizando-se régua milimetrada. $\mathrm{O}$ número de inflorescências das plantas foi obtido pela contagem apenas das plantas úteis da parcela experimental. Tanto para altura de plantas quanto para número de inflorescências, as avaliações foram realizadas em datas específicas e adequadas ao comportamento das plantas no campo. Aos 56 DAA, a parte aérea das plantas de sálvia foi coletada, ensacada, lavada e encaminhada para estufa com circulação forçada de ar, regulada a $70{ }^{\circ} \mathrm{C}$, até massa constante.

A avaliação da comunidade infestante se deu pela contagem das espécies presentes na área amostral de 0,48 $\mathrm{m}^{2}$ (correspondente a todo o espaço próximo às plantas úteis da parcelas) e foi realizada aos 14, 21, 28, 42 e 56 DAA.

Os dados de densidade (número de plantas por $\mathrm{m}^{2}$ ) referentes à comunidade infestante foram representados graficamente em função dos dias após a implantação dos tratamentos.

Os dados obtidos foram submetidos à aplicação do teste $\mathrm{F}$ na análise de variância, utilizando para comparação de médias o teste de Tukey a $5 \%$ de probabilidade.

\section{RESULTADOS E DISCUSSÃO}

Para o número de inflorescências das plantas de Salvia splendens (Tabela 1), observouse que a interação entre quantidade de palha e dose de herbicida foi significativa aos 7,14 e 28 DAA.

A testemunha (sem aplicação de herbicida e sem palha) apresentou os maiores valores de número de inflorescências até os 28 DAA; a partir desse período, a interação entre os fatores foi não-significativa, não sendo observada diferença estatística entre as doses de herbicidas, independentemente da utilização e quantidade de palha.

Os tratamentos que receberam aplicação de oxyfluorfen nas doses de 1 e $2 \mathrm{~L} \mathrm{ha}^{-1}$ sem acréscimo de palha foram os que mostraram os menores valores de número de inflorescências até os 28 DAA. Já os tratamentos que receberam acréscimo de palha $\left(4,6\right.$ e 8 tha $\left.^{-1}\right)$, sem herbicida ou nas duas doses testadas, não apresentaram diferenças estatísticas no número de inflorescências.
Assim, o herbicida aplicado diretamente sobre as plantas de sálvia, sem palha, proporcionou menor número de inflorescências. A quantidade de palha aplicada não interferiu no número de inflorescências da planta, exceto para $0 \mathrm{t} \mathrm{ha}^{-1}$.

Analisando os dados de altura de planta (Tabela 2), verificou-se que houve interação significativa entre as variáveis estudadas até os 28 DAA.

Nos tratamentos sem aplicação de palha, a testemunha apresentou os maiores valores de altura de planta até os 28 DAA. A partir desse periodo, os demais tratamentos apresentaram-se semelhantes à testemunha. Naqueles que receberam aplicação de palha, nas três diferentes quantidades, não foi verificada diferença estatística entre aqueles tratamentos com ou sem aplicação do herbicida, em relação à altura das plantas.

Nas doses de 1 e $2 \mathrm{~L} \mathrm{ha}^{-1}$ do produto, observou-se, de maneira geral, que os tratamentos que não receberam aplicação de palha apresentaram menores valores de altura de planta, evidenciando a contribuição positiva da palha na altura das plantas de sálvia quando se utiliza o herbicida. Também Ronchi \& Silva (2003) verificaram que a altura de mudas de café reduziu $27 \%$ com aplicação de oxyflurfen, porém a aplicação foi total.

De forma semelhante, Azania et al. (2004) constataram que a altura de dois cultivares de amendoim foi menor nos tratamentos com ausência de palha de cana-de-açúcar quando se aplicou o herbicida imazapic.

Analisando os dados de massa seca da parte aérea de sálvia (Tabela 3), observa-se que não houve diferença estatística entre os tratamentos quando foram aplicadas diferentes quantidades de palha. Contudo, foi observada diferença quando se compararam as doses de aplicação do oxyfluorfen, evidenciando que as plantas que receberam maior dose do herbicida, independentemente da quantidade de palha aplicada, foram as que se apresentaram com maiores valores de massa seca. Esse resultado diverge do observado por Ronchi \& Silva (2003) em mudas de café, os quais verificaram que a massa seca da parte aérea foi reduzida em 44\% quando em aplicação direta de oxyfluorfen. Da mesma forma, Azania et al. (2004) 
Tabela 1 - Quadro de análise de variância referente ao número de inflorescências de sálvia (Salvia splendens) submetida ao tratamento com oxyfluorfen. Jaboticabal, 2007

\begin{tabular}{|c|c|c|c|c|c|}
\hline \multirow{2}{*}{ Variável } & \multicolumn{5}{|c|}{ Dia após o tratamento (DAA) } \\
\hline & 7 & 14 & 28 & 42 & 56 \\
\hline Palha (P) & $27,80^{* *}$ & $49,78^{* *}$ & $85,24 * *$ & $32,20 * *$ & $3,14 \mathrm{~ns}$ \\
\hline Dose (D) & $4,71^{* *}$ & $17,97^{* *}$ & $15,36 * *$ & $3,91 \mathrm{~ns}$ & $4,72 \mathrm{~ns}$ \\
\hline $\mathrm{P} \times \mathrm{D}$ & $8,13 * *$ & $13,69 * *$ & $14,89^{* *}$ & $5,99 \mathrm{~ns}$ & $3,09 \mathrm{~ns}$ \\
\hline Blocos & $0,97 \mathrm{~ns}$ & $2,50 \mathrm{~ns}$ & $1,35 \mathrm{~ns}$ & $1,84 \mathrm{~ns}$ & $0,96 \mathrm{~ns}$ \\
\hline Resíduo & 0,85 & 2,01 & 1,87 & 2,97 & 109,22 \\
\hline Dd. $0 \mathrm{t} \mathrm{ha}^{-1}$ palha & $27,45^{* *}$ & $57,07^{* *}$ & $57,86^{* *}$ & - & - \\
\hline Dd. $4 \mathrm{t} \mathrm{ha}^{-1}$ palha & $0,03 \mathrm{~ns}$ & $1,21 \mathrm{~ns}$ & $0,16 \mathrm{~ns}$ & - & - \\
\hline Dd. $6 \mathrm{t} \mathrm{ha}^{-1}$ palha & $0,23 \mathrm{~ns}$ & $0,33 \mathrm{~ns}$ & $1,65 \mathrm{~ns}$ & - & - \\
\hline Dd. $8 \mathrm{t} \mathrm{ha}^{-1}$ palha & $1,95 \mathrm{~ns}$ & $0,43 \mathrm{~ns}$ & $0,36 \mathrm{~ns}$ & - & - \\
\hline Pd. 0 $\mathrm{L} \mathrm{ha}^{-1}$ dose & $1,77 \mathrm{~ns}$ & $0,55 \mathrm{~ns}$ & $1,65 \mathrm{~ns}$ & - & - \\
\hline Pd. $1 \mathrm{~L} \mathrm{ha}^{-1}$ dose & $20,13 * *$ & $35,19 * *$ & $49,85 * *$ & - & - \\
\hline Pd. $2 \mathrm{~L} \mathrm{ha}^{-1}$ dose & $22,54 * *$ & $41,42 * *$ & $63,51 * *$ & - & - \\
\hline $\mathrm{CV} \%$ & 24,01 & 24,16 & 18,10 & 18,07 & 20,45 \\
\hline Média geral & 3,85 & 5,87 & 7,56 & 9,54 & 8,89 \\
\hline Palha $0 \mathrm{t} \mathrm{ha}^{-1}$ & $1,60 \mathrm{~b} 1$ & $2,82 \mathrm{~b}$ & $3,58 \mathrm{~b}$ & $7,13 \mathrm{~b}$ & $8,25 \mathrm{a}$ \\
\hline Palha $4 \mathrm{t} \mathrm{ha}^{-1}$ & $4,85 \mathrm{a}$ & $6,96 \mathrm{a}$ & $9,07 \mathrm{a}$ & $10,29 \mathrm{a}$ & $8,79 \mathrm{a}$ \\
\hline Palha $6 \mathrm{t} \mathrm{ha}^{-1}$ & $4,70 \mathrm{a}$ & $6,82 \mathrm{a}$ & $8,52 \mathrm{a}$ & $9,97 \mathrm{a}$ & $9,05 \mathrm{a}$ \\
\hline Palha $8 \mathrm{t} \mathrm{ha}^{-1}$ & $4,25 \mathrm{a}$ & $6,90 \mathrm{a}$ & $9,06 \mathrm{a}$ & $10,76 \mathrm{a}$ & $9,48 \mathrm{a}$ \\
\hline Dose $0 \mathrm{~L} \mathrm{ha}^{-1}$ & $4,47 \mathrm{a}$ & $7,10 \mathrm{a}$ & $8,60 \mathrm{a}$ & $9,95 \mathrm{a}$ & $8,28 \mathrm{a}$ \\
\hline Dose $1 \mathrm{~L} \mathrm{ha}^{-1}$ & $3,48 \mathrm{~b}$ & $5,34 \mathrm{~b}$ & $7,41 \mathrm{ab}$ & $9,67 \mathrm{a}$ & $9,31 \mathrm{a}$ \\
\hline Dose $2 \mathrm{~L} \mathrm{ha}^{-1}$ & $3,60 \mathrm{~b}$ & $5,19 \mathrm{~b}$ & $6,66 \mathrm{~b}$ & $8,99 \mathrm{a}$ & $9,09 \mathrm{a}$ \\
\hline \multicolumn{6}{|l|}{ Dose d. $0 \mathrm{t} \mathrm{ha}^{-1}$ palha } \\
\hline $0 \mathrm{~L} \mathrm{ha}^{-1}$ & $4,62 \mathrm{a}$ & $7,17 \mathrm{a}$ & $7,90 \mathrm{a}$ & - & - \\
\hline $1 \mathrm{~L} \mathrm{ha}^{-1}$ & $0,12 \mathrm{~b}$ & $0,92 \mathrm{~b}$ & $2,12 \mathrm{~b}$ & - & - \\
\hline $2 \mathrm{~L} \mathrm{ha}^{-1}$ & $0,50 \mathrm{~b}$ & $0,37 \mathrm{~b}$ & $0,72 \mathrm{~b}$ & - & - \\
\hline \multicolumn{6}{|l|}{ Dose d. $4 \mathrm{tha}^{-1}$ palha } \\
\hline $0 \mathrm{~L} \mathrm{ha}^{-1}$ & $4,80 \mathrm{a}$ & $7,50 \mathrm{a}$ & $8,85 \mathrm{a}$ & - & - \\
\hline $1 \mathrm{~L} \mathrm{ha}^{-1}$ & $4,80 \mathrm{a}$ & $6,40 \mathrm{a}$ & $9,25 \mathrm{a}$ & - & - \\
\hline $2 \mathrm{~L} \mathrm{ha}^{-1}$ & $4,95 \mathrm{a}$ & $7,00 \mathrm{a}$ & $9,12 \mathrm{a}$ & - & - \\
\hline \multicolumn{6}{|l|}{ Dose d.6 t ha ${ }^{-1}$ palha } \\
\hline $0 \mathrm{~L} \mathrm{ha}^{-1}$ & $4,97 \mathrm{a}$ & $7,12 \mathrm{a}$ & $8,30 \mathrm{a}$ & - & - \\
\hline $1 \mathrm{~L} \mathrm{ha}^{-1}$ & $4,60 \mathrm{a}$ & $6,80 \mathrm{a}$ & $9,25 \mathrm{a}$ & - & - \\
\hline $2 \mathrm{~L} \mathrm{ha}^{-1}$ & $4,52 \mathrm{a}$ & $6,55 \mathrm{a}$ & $8,02 \mathrm{a}$ & - & - \\
\hline \multicolumn{6}{|l|}{ Dose d. $8 \mathrm{t} \mathrm{ha}^{-1}$ palha } \\
\hline $0 \mathrm{~L} \mathrm{ha}^{-1}$ & $3,50 \mathrm{a}$ & $6,60 \mathrm{a}$ & $9,37 \mathrm{a}$ & - & - \\
\hline $1 \mathrm{~L} \mathrm{ha}^{-1}$ & $4,40 \mathrm{a}$ & $7,25 \mathrm{a}$ & $9,05 \mathrm{a}$ & - & - \\
\hline $2 \mathrm{~L} \mathrm{ha}^{-1}$ & $4,87 \mathrm{a}$ & $6,85 \mathrm{a}$ & $8,77 \mathrm{a}$ & - & - \\
\hline \multicolumn{6}{|l|}{ Palha d. 0 $\mathrm{L} \mathrm{ha}^{-1}$ dose } \\
\hline $0 \mathrm{t} \mathrm{ha}^{-1}$ & $4,62 \mathrm{a}$ & $7,17 \mathrm{a}$ & $7,90 \mathrm{a}$ & - & - \\
\hline $4 \mathrm{tha}^{-1}$ & $4,80 \mathrm{a}$ & $7,50 \mathrm{a}$ & $8,85 \mathrm{a}$ & - & - \\
\hline $6 \mathrm{tha}^{-1}$ & $4,97 \mathrm{a}$ & $7,12 \mathrm{a}$ & $8,30 \mathrm{a}$ & - & - \\
\hline $8 \mathrm{t} \mathrm{ha}^{\mathrm{l}}$ & $3,50 \mathrm{a}$ & $6,60 \mathrm{a}$ & $9,37 \mathrm{a}$ & - & - \\
\hline \multicolumn{6}{|l|}{ Palha d. $1 \mathrm{~L} \mathrm{ha}^{-1}$ dose } \\
\hline $0 \mathrm{tha}^{-1}$ & $0,12 \mathrm{~b}$ & $0,92 \mathrm{~b}$ & $2,12 \mathrm{~b}$ & - & - \\
\hline $4 \mathrm{tha}^{-1}$ & $4,80 \mathrm{a}$ & $6,40 \mathrm{a}$ & $9,25 \mathrm{a}$ & - & - \\
\hline $6 \mathrm{tha}^{-1}$ & $4,60 \mathrm{a}$ & $6,80 \mathrm{a}$ & $9,25 \mathrm{a}$ & - & - \\
\hline $8 \mathrm{tha}^{-1}$ & $4,40 \mathrm{a}$ & $7,25 \mathrm{a}$ & $9,05 \mathrm{a}$ & - & - \\
\hline \multicolumn{6}{|l|}{ Palha d. $2 \mathrm{~L} \mathrm{ha}^{-1}$ dose } \\
\hline $0 \mathrm{tha}^{-1}$ & $0,05 \mathrm{~b}$ & $0,37 \mathrm{~b}$ & $0,72 \mathrm{~b}$ & - & - \\
\hline $4 \mathrm{tha}^{-1}$ & $4,95 \mathrm{a}$ & $7,00 \mathrm{a}$ & $9,12 \mathrm{a}$ & - & - \\
\hline $6 \mathrm{tha}^{-1}$ & $4,52 \mathrm{a}$ & $6,55 \mathrm{a}$ & $8,02 \mathrm{a}$ & - & - \\
\hline $8 \mathrm{tha}^{-1}$ & $4,87 \mathrm{a}$ & $6,85 \mathrm{a}$ & $8,77 \mathrm{a}$ & - & - \\
\hline DMS palha & 1,02 & 1,57 & 1,51 & 1,90 & 2,01 \\
\hline DMS dose & 0,80 & 1,23 & 1,18 & 1,49 & 1,57 \\
\hline
\end{tabular}

ns - não-significativo; *; ** significativos a 5 e $1 \%$ de probabilidade, respectivamente; 1 - valores acompanhados de mesma letra na coluna e para a mesma variável não diferem entre si estatisticamente pelo teste de Tukey (5\%); DMS - diferença mínima significativa. 
Tabela 2 - Quadro de análise de variância referente à altura de sálvia (Salvia splendens) submetida ao tratamento com oxyfluorfen. Jaboticabal, 2007

\begin{tabular}{|c|c|c|c|c|c|c|}
\hline \multirow{2}{*}{ Variável } & \multicolumn{6}{|c|}{ Dia após a aplicação (DAA) } \\
\hline & 7 & 14 & 21 & 28 & 42 & 56 \\
\hline Palha (P) & $17,18^{*}$ & $70,72 * *$ & $99,24 * *$ & $152,52 * *$ & $15,02^{\mathrm{ns}}$ & $19,05^{\mathrm{ns}}$ \\
\hline Dose (D) & $8,33^{\mathrm{ns}}$ & $3,34 *$ & $19,77^{\mathrm{ns}}$ & $15,89^{\mathrm{ns}}$ & $3,25^{\mathrm{ns}}$ & $2,02^{\mathrm{ns}}$ \\
\hline $\mathrm{P} \times \mathrm{D}$ & $12,50 *$ & $3,23 *$ & $27,24 * *$ & $29,59 *$ & $37,36^{\mathrm{ns}}$ & $76,74^{\mathrm{ns}}$ \\
\hline Blocos & $19,96 * *$ & $21,55^{*}$ & $26,07 *$ & $21,41^{\mathrm{ns}}$ & $7,47^{\mathrm{ns}}$ & $69,94^{\mathrm{ns}}$ \\
\hline Resíduo & 4,05 & 6,73 & 7,71 & 11,53 & 21,36 & 32,39 \\
\hline Dd. $0 \mathrm{t} \mathrm{ha}^{-1}$ palha & $33,33 * *$ & $79,00 * *$ & $86,33 * *$ & $95,08 * *$ & - & - \\
\hline Dd. $4 \mathrm{t} \mathrm{ha}^{-1}$ palha & $2,08^{\mathrm{ns}}$ & $4,08^{\mathrm{ns}}$ & $8,58^{\mathrm{ns}}$ & $0,75^{\mathrm{ns}}$ & - & - \\
\hline Dd. $6 \mathrm{t} \mathrm{ha}^{-1}$ palha & $2,08^{\mathrm{ns}}$ & $0,75^{\mathrm{ns}}$ & $1,33^{\mathrm{ns}}$ & $0,25^{\mathrm{ns}}$ & - & - \\
\hline Dd. $8 \mathrm{t} \mathrm{ha}^{-1}$ palha & $8,33^{\mathrm{ns}}$ & $4,08^{\mathrm{ns}}$ & $5,25^{\mathrm{ns}}$ & $8,58^{\text {ns }}$ & - & - \\
\hline Pd. $0 \mathrm{~L} \mathrm{ha}^{-1}$ dose & $5,72^{\mathrm{ns}}$ & $1,83^{\mathrm{ns}}$ & $3,56^{\mathrm{ns}}$ & $3,22^{\mathrm{ns}}$ & - & - \\
\hline Pd. $1 \mathrm{~L} \mathrm{ha}^{-1}$ dose & $2,43^{\mathrm{ns}}$ & $58,56 * *$ & $79,16 * *$ & $112,41 * *$ & - & - \\
\hline Pd. $2 \mathrm{~L} \mathrm{ha}^{-1}$ dose & $26,56 * *$ & $53,89 * *$ & $71,00 * *$ & $96,06 * *$ & - & - \\
\hline $\mathrm{CV} \%$ & 6,09 & 7,16 & 7,36 & 8,55 & 10,35 & 11,34 \\
\hline Média geral & 33,02 & 36,25 & 37,72 & 39,70 & 44,62 & 50,16 \\
\hline Palha $0 \mathrm{t} \mathrm{ha}^{-1}$ & 31,66 b 1 & $32,75 \mathrm{~b}$ & $33,41 \mathrm{~b}$ & $34,41 \mathrm{~b}$ & $43,08 \mathrm{a}$ & $51,66 \mathrm{a}$ \\
\hline Palha $4 \mathrm{t} \mathrm{ha}^{-1}$ & $32,91 \mathrm{ab}$ & $37,66 \mathrm{a}$ & $39,16 \mathrm{a}$ & $41,75 \mathrm{a}$ & $45,16 \mathrm{a}$ & $50,75 \mathrm{a}$ \\
\hline Palha $6 \mathrm{t} \mathrm{ha}^{-1}$ & $32,91 \mathrm{ab}$ & $36,50 \mathrm{a}$ & $39,08 \mathrm{a}$ & $40,75 \mathrm{a}$ & $44,58 \mathrm{a}$ & $49,16 \mathrm{a}$ \\
\hline Palha $8 \mathrm{t} \mathrm{ha}^{-1}$ & $34,58 \mathrm{a}$ & $38,08 \mathrm{a}$ & $39,25 \mathrm{a}$ & $41,91 \mathrm{a}$ & $45,66 \mathrm{a}$ & $49,08 \mathrm{a}$ \\
\hline Dose $0 \mathrm{~L} \mathrm{ha}^{-1}$ & $33,43 \mathrm{a}$ & $37,25 \mathrm{a}$ & $38,93 \mathrm{a}$ & $40,81 \mathrm{a}$ & $45,00 \mathrm{a}$ & $50,56 \mathrm{a}$ \\
\hline Dose $1 \mathrm{~L} \mathrm{ha}^{-1}$ & $32,18 \mathrm{a}$ & $34,93 \mathrm{~b}$ & $37,50 \mathrm{a}$ & $38,87 \mathrm{a}$ & $44,12 \mathrm{a}$ & $49,87 \mathrm{a}$ \\
\hline Dose $2 \mathrm{~L} \mathrm{ha}^{-1}$ & $33,43 \mathrm{a}$ & $36,56 \mathrm{ab}$ & $36,75 \mathrm{a}$ & $39,43 \mathrm{a}$ & $44,75 \mathrm{a}$ & $50,06 \mathrm{a}$ \\
\hline \multicolumn{7}{|l|}{ Dose d. $0 \mathrm{t} \mathrm{ha}^{-1}$ palha } \\
\hline $0 \mathrm{~L} \mathrm{ha}^{-1}$ & $35,00 \mathrm{a}$ & $37,75 \mathrm{a}$ & $38,75 \mathrm{a}$ & $40,00 \mathrm{a}$ & - & - \\
\hline $1 \mathrm{~L} \mathrm{ha}^{-1}$ & $30,00 \mathrm{~b}$ & $29,25 \mathrm{~b}$ & $30,25 \mathrm{~b}$ & $31,00 \mathrm{~b}$ & - & - \\
\hline $2 \mathrm{~L} \mathrm{ha}^{-1}$ & $30,00 \mathrm{~b}$ & $31,25 \mathrm{~b}$ & $31,25 \mathrm{~b}$ & $32,25 \mathrm{~b}$ & - & - \\
\hline \multicolumn{7}{|l|}{ Dose d. $4 \mathrm{t} \mathrm{ha}^{-1}$ palha } \\
\hline $0 \mathrm{~L} \mathrm{ha}^{-1}$ & $32,50 \mathrm{a}$ & $37,50 \mathrm{a}$ & $40,25 \mathrm{a}$ & $42,00 \mathrm{a}$ & - & - \\
\hline $1 \mathrm{~L} \mathrm{ha}^{-1}$ & $32,50 \mathrm{a}$ & $36,75 \mathrm{a}$ & $37,50 \mathrm{a}$ & $41,25 \mathrm{a}$ & - & - \\
\hline $2 \mathrm{~L} \mathrm{ha}^{-1}$ & $33,75 \mathrm{a}$ & $38,75 \mathrm{a}$ & $39,75 \mathrm{a}$ & $42,00 \mathrm{a}$ & - & - \\
\hline \multicolumn{7}{|l|}{ Dose d. $6 \mathrm{t} \mathrm{ha}^{-1}$ palha } \\
\hline $0 \mathrm{~L} \mathrm{ha}^{-1}$ & $32,50 \mathrm{a}$ & $36,25 \mathrm{a}$ & $38,75 \mathrm{a}$ & $41,00 \mathrm{a}$ & - & - \\
\hline $1 \mathrm{~L} \mathrm{ha}^{-1}$ & $32,50 \mathrm{a}$ & $36,25 \mathrm{a}$ & $39,75 \mathrm{a}$ & $40,75 \mathrm{a}$ & - & - \\
\hline $2 \mathrm{~L} \mathrm{ha}^{-1}$ & $33,75 \mathrm{a}$ & $37,00 \mathrm{a}$ & $38,75 \mathrm{a}$ & $40,50 \mathrm{a}$ & - & - \\
\hline \multicolumn{7}{|l|}{ Dose d. $8 \mathrm{t} \mathrm{ha}^{-1}$ palha } \\
\hline $0 \mathrm{~L} \mathrm{ha}^{-1}$ & $33,75 \mathrm{a}$ & $37,50 \mathrm{a}$ & $38,00 \mathrm{a}$ & $40,25 \mathrm{a}$ & - & - \\
\hline $1 \mathrm{~L} \mathrm{ha}^{-1}$ & $33,75 \mathrm{a}$ & $37,50 \mathrm{a}$ & $39,50 \mathrm{a}$ & $42,50 \mathrm{a}$ & - & - \\
\hline $2 \mathrm{~L} \mathrm{ha}^{-1}$ & $36,25 \mathrm{a}$ & $39,25 \mathrm{a}$ & $40,25 \mathrm{a}$ & $43,00 \mathrm{a}$ & - & - \\
\hline \multicolumn{7}{|l|}{ Palha d. $0 \mathrm{~L} \mathrm{ha}^{-1}$ dose } \\
\hline $0 \mathrm{t} \mathrm{ha}^{-1}$ & $35,00 \mathrm{a}$ & $37,75 \mathrm{a}$ & $38,75 \mathrm{a}$ & $40,00 \mathrm{a}$ & - & - \\
\hline $4 \mathrm{t} \mathrm{ha}^{-1}$ & $32,50 \mathrm{a}$ & $37,75 \mathrm{a}$ & $40,25 \mathrm{a}$ & $42,00 \mathrm{a}$ & - & - \\
\hline $6 \mathrm{tha}^{-1}$ & $32,50 \mathrm{a}$ & $36,25 \mathrm{a}$ & $38,75 \mathrm{a}$ & $41,00 \mathrm{a}$ & - & - \\
\hline $8 \mathrm{tha}^{-1}$ & $33,75 \mathrm{a}$ & $37,50 \mathrm{a}$ & $38,00 \mathrm{a}$ & $40,25 \mathrm{a}$ & - & - \\
\hline \multicolumn{7}{|l|}{ Palha d. $1 \mathrm{~L} \mathrm{ha}^{-1}$ dose } \\
\hline $0 \mathrm{t} \mathrm{ha}^{-1}$ & $30,00 \mathrm{a}$ & $29,50 \mathrm{~b}$ & $30,25 \mathrm{~b}$ & $31,00 \mathrm{~b}$ & - & - \\
\hline $4 \mathrm{tha}^{-1}$ & $32,50 \mathrm{a}$ & $36,75 \mathrm{a}$ & $37,50 \mathrm{a}$ & $41,25 \mathrm{a}$ & - & - \\
\hline $6 \mathrm{tha}^{-1}$ & $32,50 \mathrm{a}$ & $36,25 \mathrm{a}$ & $39,75 \mathrm{a}$ & $40,75 \mathrm{a}$ & - & - \\
\hline $8 \mathrm{t} \mathrm{ha}^{-1}$ & $33,75 \mathrm{a}$ & $37,50 \mathrm{a}$ & $39,50 \mathrm{a}$ & $42,50 \mathrm{a}$ & - & - \\
\hline \multicolumn{7}{|l|}{ Palha d. $2 \mathrm{~L} \mathrm{ha}^{-1}$ dose } \\
\hline $0 \mathrm{t} \mathrm{ha}^{-1}$ & $30,00 \mathrm{~b}$ & $31,25 \mathrm{~b}$ & $31,25 \mathrm{~b}$ & $32,25 \mathrm{~b}$ & - & - \\
\hline $4 \mathrm{tha}^{-1}$ & $33,75 \mathrm{ab}$ & $38,75 \mathrm{a}$ & $39,75 \mathrm{a}$ & $42,00 \mathrm{a}$ & - & - \\
\hline $6 \mathrm{tha}^{-1}$ & $33,75 \mathrm{ab}$ & $37,00 \mathrm{a}$ & $38,75 \mathrm{a}$ & $40,50 \mathrm{a}$ & - & - \\
\hline $8 \mathrm{tha}^{-1}$ & $36,25 \mathrm{a}$ & $39,25 \mathrm{a}$ & $40,25 \mathrm{a}$ & $43,00 \mathrm{a}$ & - & - \\
\hline DMS palha & 2,22 & 2,86 & 3,06 & 3,75 & 5,10 & 6,29 \\
\hline DMS dose & 1,74 & 2,25 & 2.41 & 2,94 & 4,01 & 4,94 \\
\hline
\end{tabular}

ns - não-significativo; *; ** significativos a 5 e $1 \%$ de probabilidade, respectivamente; 1 - valores acompanhados de mesma letra na coluna e para a mesma variável não diferem entre si estatisticamente pelo teste de Tukey (5\%); DMS - diferença mínima significativa. 
Tabela 3 - Quadro de análise de variância referente à massa seca da parte aérea, em gramas, de sálvia (Salvia splendens) submetida ao tratamento com oxyfluorfen. Jaboticabal, 2007

\begin{tabular}{|c|c|}
\hline Variável & Massa seca \\
\hline Palha (P) & $229,80 \mathrm{~ns}$ \\
\hline Dose (D) & $1000,07 *$ \\
\hline $\mathrm{P} \times \mathrm{D}$ & $125,75 \mathrm{~ns}$ \\
\hline Blocos & $732,58 \mathrm{~ns}$ \\
\hline Resíduo & 255,95 \\
\hline CV\% & 27,71 \\
\hline Média geral & 57,71 \\
\hline Palha $0 \mathrm{t} \mathrm{ha}^{-1}$ & 57,88 a 1 \\
\hline Palha 4 t ha $^{-1}$ & $62,90 \mathrm{a}$ \\
\hline Palha $6 \mathrm{t} \mathrm{ha}^{-1}$ & $52,19 \mathrm{a}$ \\
\hline Palha 8 t ha $^{-1}$ & $57,87 \mathrm{a}$ \\
\hline Dose $0 \mathrm{~L} \mathrm{ha}^{-1}$ & $49,11 \mathrm{~b}$ \\
\hline Dose $1 \mathrm{~L} \mathrm{ha}^{-1}$ & $59,37 \mathrm{ab}$ \\
\hline Dose $2 \mathrm{~L} \mathrm{ha}^{-1}$ & $64,66 \mathrm{a}$ \\
\hline DMS palha & 17,67 \\
\hline DMS dose & 13,88 \\
\hline $\begin{array}{l}\text { ns - não-significat } \\
\text { probabilidade, respec } \\
\text { mesma letra na coluna } \\
\text { si estatisticamente pe } \\
\text { mínima significativa. }\end{array}$ & $\begin{array}{l}\text { ficativos a } 5 \text { e } 1 \% \text { de } \\
\text { valores acompanhados de } \\
\text { variável não diferem entre } \\
(5 \%) \text { DMS - diferença }\end{array}$ \\
\hline
\end{tabular}

verificaram que plantas de amendoim, quando submetidas à aplicação direta de imazapic, sem acréscimo de palha de cana-de-açúcar, mostraram menores valores de massa seca.

Analisando os dados dos efeitos fitotóxicos (Tabela 4), observou-se que somente aos 56 DAA a interação entre as variáveis estudadas foi não-significativa.

A pulverização em pré-emergência das plantas daninhas, em aplicação dirigida de ambas as doses de oxyfluorfen, na ausência da camada de palha, causou injúrias nas plantas de sálvia em todos os periodos avaliados, exceto aos 56 DAA. Observaram-se, ainda, maiores efeitos fitotóxicos aos 21 e 28 DAA nos tratamentos que receberam maior dose do herbicida. As plantas de sálvia tiveram queda total e/ou parcial das folhas e flores, assim como necrosamento de folhas. No entanto, Alves et al. (2000) comentam que os efeitos fitotóxicos observados para este herbicida ficam restritos aos locais de contato entre o produto e a planta, não havendo evolução dos efeitos com o desenvolvimento das plantas. Mesmo assim, o que se busca neste trabalho é o efeito visual, que não pode ser comprometido nem pelas injúrias nas plantas nem pela presença de plantas daninhas.

Em mudas de café, Ronchi \& Silva (2003) observaram que o herbicida oxyfluorfen, entre outros, aplicado em pós-emergência diretamente nas plantas causou severos efeitos fitotóxicos, caracterizados por queimaduras e deformações em toda a lâmina foliar atingida pelo herbicida. Igualmente, Freitas et al. (2007b) verificaram que o herbicida oxyfuorfen aplicado diretamente nas mudas das plantas ornamentais estrelitzia (Strelitzia reginae), copo-deleite (Zantedeschia aethiopica) e palmeiraaustraliana (Archonotphoenix cunninghamiana) provocou sintomas fitotóxicos, sendo mais severo em palmeira-australiana, caracterizado por necrose nas folhas mais novas; contudo, em aplicação dirigida, não foram verificados efeitos fitotóxicos em nenhuma espécie. Também Freitas et al. (2007a) não observaram efeitos fitotóxicos de oxyfluorfen quando foi realizada aplicação dirigida em orquídeas (Epidendrum ibaguensis e Dendrobium sp.)

O herbicida oxyfluorfen, veiculado à palha de arroz, proporcionou menores niveis de injúrias nas plantas de sálvia, possivelmente devido à retenção e lenta liberação do produto na camada de palha. O mesmo comportamento foi observado por Azania et al. (2004) na cultura do amendoim, quanto aos efeitos fitotóxicos do herbicida imazapic.

A nota média de fitotoxicidade, aos 7,14 , $21,28,35$ e 42 DAA, nos tratamentos que receberam o herbicida veiculado à palha foi 2 , correspondendo a sintomas classificados na variação de muito leves a leves. Já os tratamentos que receberam pulverização direta do herbicida, sem a presença da palha, tiveram nota média de 5 , exceto aos 42 DAA, caracterizando os sintomas que aparentam causar efeitos fitotóxicos quase forte a forte.

A comunidade infestante observada e levantada no experimento foi composta por 25 espécies de plantas daninhas, evidenciando o aparecimento de espécies diferentes das que foram introduzidas na área. Dentre as dicotiledôneas, destacaram-se, em número de espécies, as famílias Euphorbiaceae e 
Tabela 4 - Quadro de análise de variância referente às notas de fitotoxicidade de sálvia (Salvia splendens) submetida ao tratamento com oxyfluorfen. Jaboticabal, 2007

\begin{tabular}{|c|c|c|c|c|c|c|c|}
\hline \multirow{2}{*}{ Variável } & \multicolumn{7}{|c|}{ Dia após a aplicação (DAA) } \\
\hline & 7 & 14 & 21 & 28 & 35 & 42 & 56 \\
\hline Palha (P) & $61,43 * *$ & $34,23 * *$ & $34,57 * *$ & $43,30 * *$ & $42,58 * *$ & $2,80^{\mathrm{ns}}$ & $1,88^{\mathrm{ns}}$ \\
\hline Dose (D) & $107,39 * *$ & $44,68 * *$ & $78,83 * *$ & $67,15 * *$ & $58,67 * *$ & $73,27 * *$ & $44,28 * *$ \\
\hline $\mathrm{P} \times \mathrm{D}$ & $15,92 * *$ & $11,53 * *$ & $10,21 * *$ & $12,84 * *$ & $11,10 * *$ & $2,96 *$ & $0,54^{\mathrm{ns}}$ \\
\hline Blocos & $1,42^{\mathrm{ns}}$ & $0,32^{\mathrm{ns}}$ & $0,52^{\mathrm{ns}}$ & $0,07^{\mathrm{ns}}$ & $1,53^{\mathrm{ns}}$ & $1,94^{\mathrm{ns}}$ & $3,15 *$ \\
\hline Resíduo & 0,24 & 0,40 & 0,31 & 0,36 & 0,45 & 0,12 & 0,21 \\
\hline Dd. $0 \mathrm{t} \mathrm{ha}^{-1}$ palha & $122,25 * *$ & $69,19 * *$ & $82,76 * *$ & $87,46 * *$ & $75,95 * *$ & $20,05 * *$ & - \\
\hline Dd. $4 \mathrm{t} \mathrm{ha}^{-1}$ palha & $8,39 * *$ & $4,32 *$ & $6,54 * *$ & $6,23 * *$ & $2,39^{\mathrm{ns}}$ & $23,94 * *$ & - \\
\hline Dd. $6 \mathrm{t} \mathrm{ha}^{-1}$ palha & $12,09 * *$ & $4,32 *$ & $12,83 * *$ & $7,15 * *$ & $9,03 * *$ & $27,82 * *$ & - \\
\hline Dd. $8 \mathrm{t} \mathrm{ha}^{-1}$ palha & $12,42 * *$ & $1,44^{\mathrm{ns}}$ & $7,33^{* *}$ & $4,84 *$ & $4,60 *$ & $10,35 * *$ & - \\
\hline Pd. $0 \mathrm{~L} \mathrm{ha}^{-1}$ dose & $0,00^{\mathrm{ns}}$ & $0,56^{\mathrm{ns}}$ & $0,00^{\mathrm{ns}}$ & $0,00^{\mathrm{ns}}$ & $0,00^{\mathrm{ns}}$ & $0,00^{\mathrm{ns}}$ & - \\
\hline Pd. 1 L ha ${ }^{-1}$ dose & $43,24 * *$ & $21,98 * *$ & $15,97 * *$ & $20,76^{* *}$ & $26,31 * *$ & $4,36^{*}$ & - \\
\hline Pd. $2 \mathrm{~L} \mathrm{ha}^{-1}$ dose & $50,04 * *$ & $34,75 * *$ & $39,02 * *$ & $48,23 * *$ & $38,48 * *$ & $4,36 * *$ & - \\
\hline $\mathrm{CV} \%$ & 20,09 & 26,55 & 23,34 & 25,30 & 27,34 & 19,13 & 24,61 \\
\hline Média geral & 2,47 & 2,39 & 2,41 & 2,37 & 2,45 & 1,87 & 1,89 \\
\hline Palha $0 \mathrm{t} \mathrm{ha}^{-1}$ & 4,16 a 1 & $4,00 \mathrm{a}$ & $3,83 \mathrm{a}$ & $4,08 \mathrm{a}$ & $4,33 \mathrm{a}$ & $1,91 \mathrm{ab}$ & $2,00 \mathrm{a}$ \\
\hline Palha $4 \mathrm{t} \mathrm{ha}^{-1}$ & $1,83 \mathrm{~b}$ & $1,75 \mathrm{~b}$ & $1,83 \mathrm{~b}$ & $1,75 \mathrm{~b}$ & $1,58 \mathrm{~b}$ & $1,83 \mathrm{ab}$ & $1,83 \mathrm{a}$ \\
\hline Palha $6 \mathrm{t} \mathrm{ha}^{-1}$ & $2,00 \mathrm{~b}$ & $2,00 \mathrm{~b}$ & $2,16 \mathrm{~b}$ & $1,91 \mathrm{~b}$ & $2,08 \mathrm{~b}$ & $2,08 \mathrm{a}$ & $2,08 \mathrm{a}$ \\
\hline Palha $8 \mathrm{t} \mathrm{ha}^{-1}$ & $1,91 \mathrm{~b}$ & $1,83 \mathrm{~b}$ & $1,83 \mathrm{~b}$ & $1,75 \mathrm{~b}$ & $1,83 \mathrm{~b}$ & $1,66 \mathrm{~b}$ & $1,66 \mathrm{a}$ \\
\hline Dose $0 \mathrm{~L} \mathrm{ha}^{-1}$ & $1,00 \mathrm{~b}$ & $1,18 \mathrm{~b}$ & $1,00 \mathrm{c}$ & $1,00 \mathrm{c}$ & $1,00 \mathrm{~b}$ & $1,00 \mathrm{~b}$ & $1,00 \mathrm{~b}$ \\
\hline Dose $1 \mathrm{~L} \mathrm{ha}^{-1}$ & $3,06 \mathrm{a}$ & $2,81 \mathrm{a}$ & $2,87 \mathrm{~b}$ & $2,75 \mathrm{~b}$ & $2,93 \mathrm{a}$ & $2,18 \mathrm{a}$ & $2,37 \mathrm{a}$ \\
\hline Dose $2 \mathrm{~L} \mathrm{ha}^{-1}$ & $3,37 \mathrm{a}$ & $3,18 \mathrm{a}$ & $3,37 \mathrm{a}$ & $3,37 \mathrm{a}$ & $3,43 \mathrm{a}$ & $2,43 \mathrm{a}$ & $2,31 \mathrm{a}$ \\
\hline \multicolumn{8}{|l|}{ Dose d. 0 t ha ${ }^{-1}$ palha } \\
\hline $0 \mathrm{~L} \mathrm{ha}^{-1}$ & $1,00 \mathrm{~b}$ & $1,00 \mathrm{~b}$ & $1,00 \mathrm{c}$ & $1,00 \mathrm{c}$ & $1,00 \mathrm{~b}$ & $1,00 \mathrm{~b}$ & - \\
\hline $1 \mathrm{~L} \mathrm{ha}^{-1}$ & $5,50 \mathrm{a}$ & $5,00 \mathrm{a}$ & $4,50 \mathrm{~b}$ & $4,75 \mathrm{~b}$ & $5,50 \mathrm{a}$ & $2,50 \mathrm{a}$ & - \\
\hline $2 \mathrm{~L} \mathrm{ha}^{-1}$ & $6,00 \mathrm{a}$ & $6,00 \mathrm{a}$ & $6,00 \mathrm{a}$ & $6,50 \mathrm{a}$ & $6,50 \mathrm{a}$ & $2,25 \mathrm{a}$ & - \\
\hline \multicolumn{8}{|l|}{ Dose d. $4 \mathrm{t} \mathrm{ha}^{-1}$ palha } \\
\hline $0 \mathrm{~L} \mathrm{ha}^{-1}$ & $1,00 \mathrm{~b}$ & $1,00 \mathrm{~b}$ & $1,00 \mathrm{~b}$ & $1,00 \mathrm{~b}$ & $1,00 \mathrm{a}$ & $1,00 \mathrm{c}$ & - \\
\hline $1 \mathrm{~L}$ ha- 1 & $2,25 \mathrm{a}$ & $2,00 \mathrm{ab}$ & $2,25 \mathrm{a}$ & $1,75 \mathrm{ab}$ & $1,75 \mathrm{a}$ & $1,75 \mathrm{~b}$ & - \\
\hline $2 \mathrm{~L} \mathrm{ha}^{-1}$ & $2,25 \mathrm{a}$ & $2,25 \mathrm{a}$ & $2,25 \mathrm{a}$ & $2,50 \mathrm{a}$ & $2,00 \mathrm{a}$ & $2,75 \mathrm{a}$ & - \\
\hline \multicolumn{8}{|l|}{ Dose d. $6 \mathrm{t} \mathrm{ha}^{-1}$ palha } \\
\hline $0 \mathrm{~L} \mathrm{ha}^{-1}$ & $1,00 \mathrm{~b}$ & $1,25 \mathrm{~b}$ & $1,00 \mathrm{~b}$ & $1,00 \mathrm{~b}$ & $1,00 \mathrm{~b}$ & $1,00 \mathrm{~b}$ & - \\
\hline $1 \mathrm{~L} \mathrm{ha}^{-1}$ & $2,50 \mathrm{a}$ & $2,50 \mathrm{a}$ & $2,70 \mathrm{a}$ & $2,50 \mathrm{a}$ & $2,25 \mathrm{a}$ & $2,50 \mathrm{a}$ & - \\
\hline $2 \mathrm{~L} \mathrm{ha}^{-1}$ & $2,50 \mathrm{a}$ & $2,25 \mathrm{ab}$ & $2,75 \mathrm{a}$ & $2,25 \mathrm{a}$ & $3,00 \mathrm{a}$ & $2,75 \mathrm{a}$ & - \\
\hline \multicolumn{8}{|l|}{ Dose d. $8 \mathrm{t} \mathrm{ha}^{-1}$ palha } \\
\hline $0 \mathrm{~L} \mathrm{ha}^{-1}$ & $1,00 \mathrm{~b}$ & $1,50 \mathrm{a}$ & $1,00 \mathrm{~b}$ & $1,00 \mathrm{~b}$ & $1,00 \mathrm{~b}$ & $1,00 \mathrm{~b}$ & - \\
\hline $1 \mathrm{~L} \mathrm{ha}^{-1}$ & $2,00 \mathrm{~b}$ & $1,75 \mathrm{a}$ & $2,00 \mathrm{a}$ & $2,00 \mathrm{ab}$ & $2,25 \mathrm{a}$ & $2,00 \mathrm{a}$ & - \\
\hline $2 \mathrm{~L} \mathrm{ha}^{-1}$ & $2,75 \mathrm{a}$ & $2,25 \mathrm{a}$ & $2,50 \mathrm{a}$ & $2,25 \mathrm{a}$ & $2,25 \mathrm{a}$ & $2,00 \mathrm{a}$ & - \\
\hline \multicolumn{8}{|l|}{ Palha d. 0 L ha ${ }^{-1}$ dose } \\
\hline $0 \mathrm{tha}^{-1}$ & $1,00 \mathrm{a}$ & $1,00 \mathrm{a}$ & $1,00 \mathrm{a}$ & $1,00 \mathrm{a}$ & $1,00 \mathrm{a}$ & $1,00 \mathrm{a}$ & - \\
\hline $4 \mathrm{tha}^{-1}$ & $1,00 \mathrm{a}$ & $1,00 \mathrm{a}$ & $1,00 \mathrm{a}$ & $1,00 \mathrm{a}$ & $1,00 \mathrm{a}$ & $1,00 \mathrm{a}$ & - \\
\hline $6 \mathrm{tha}^{-1}$ & $1,00 \mathrm{a}$ & $1,25 \mathrm{a}$ & $1,00 \mathrm{a}$ & $1,00 \mathrm{a}$ & $1,00 \mathrm{a}$ & $1,00 \mathrm{a}$ & - \\
\hline $8 \mathrm{tha}^{-1}$ & $1,00 \mathrm{a}$ & $1,50 \mathrm{a}$ & $1,00 \mathrm{a}$ & $1,00 \mathrm{a}$ & $1,00 \mathrm{a}$ & $1,00 \mathrm{a}$ & - \\
\hline \multicolumn{8}{|l|}{ Palha d. 1 L ha ${ }^{-1}$ dose } \\
\hline $0 \mathrm{tha}^{-1}$ & $5,50 \mathrm{a}$ & $5,00 \mathrm{a}$ & $4,50 \mathrm{a}$ & $4,75 \mathrm{a}$ & $5,50 \mathrm{a}$ & $2,50 \mathrm{a}$ & - \\
\hline $4 \mathrm{tha}^{-1}$ & $2,25 \mathrm{~b}$ & $2,00 \mathrm{~b}$ & $2,25 \mathrm{~b}$ & $1,75 \mathrm{~b}$ & $1,75 \mathrm{~b}$ & $1,75 \mathrm{~b}$ & - \\
\hline $6 \mathrm{tha}^{-1}$ & $2,50 \mathrm{~b}$ & $2,50 \mathrm{~b}$ & $2,75 \mathrm{~b}$ & $2,50 \mathrm{~b}$ & $2,25 \mathrm{~b}$ & $2,50 \mathrm{a}$ & - \\
\hline $8 \mathrm{tha}^{-1}$ & $2,00 \mathrm{~b}$ & $1,75 \mathrm{~b}$ & $2,00 \mathrm{~b}$ & $2,00 \mathrm{~b}$ & $2,25 \mathrm{~b}$ & $2,00 \mathrm{ab}$ & - \\
\hline \multicolumn{8}{|l|}{ Palha d. $2 \mathrm{~L} \mathrm{ha}^{-1}$ dose } \\
\hline $0 \mathrm{t} \mathrm{ha}^{-1}$ & $6,00 \mathrm{a}$ & $6,00 \mathrm{a}$ & $6,00 \mathrm{a}$ & $6,50 \mathrm{a}$ & $6,50 \mathrm{a}$ & $2,25 \mathrm{ab}$ & - \\
\hline $4 \mathrm{tha}^{-1}$ & $2,25 \mathrm{~b}$ & $2,25 \mathrm{~b}$ & $2,25 \mathrm{~b}$ & $2,50 \mathrm{~b}$ & $2,00 \mathrm{~b}$ & $2,75 \mathrm{a}$ & - \\
\hline $6 \mathrm{tha}^{-1}$ & $2,50 \mathrm{~b}$ & $2,25 \mathrm{~b}$ & $2,75 \mathrm{~b}$ & $2,25 \mathrm{~b}$ & $3,00 \mathrm{~b}$ & $2,75 \mathrm{a}$ & - \\
\hline $8 \mathrm{tha}^{-1}$ & $2,75 \mathrm{~b}$ & $2,25 \mathrm{~b}$ & $2,50 \mathrm{~b}$ & $2,25 \mathrm{~b}$ & $2,25 \mathrm{~b}$ & $2,00 \mathrm{~b}$ & - \\
\hline DMS palha & 0,86 & 1,10 & 0,97 & 1,04 & 1,16 & 0,62 & 0,63 \\
\hline DMS dose & 0,95 & 1,21 & 1,07 & 1,15 & 1,28 & 0,68 & 0,50 \\
\hline
\end{tabular}

ns - não-significativo; *; ** significativos a 5 e $1 \%$ de probabilidade, respectivamente; 1 - valores acompanhados de mesma letra na coluna e para a mesma variável não diferem entre si estatisticamente pelo teste de Tukey (5\%); DMS - diferença mínima significativa. 
Leguminosae - subfamília Papilionoideae, ambas com três espécies de plantas daninhas, seguidas pelas familias Amaranthaceae e Asteraceae, com duas espécies. Dentre as espécies monocotiledôneas, uma pertence à familia Commelinaceae, duas à familia Cyperaceae e cinco à familia Gramineae, sendo essa, inclusive, a mais numerosa entre todas as familias de plantas daninhas presentes na área experimental, representando $20 \%$ da comunidade infestante.

Dentre as espécies de plantas daninhas encontradas na área experimental, destacaram-se braquiária (Brachiaria decumbens BRADC), caruru (Amaranthus viridis - AMAVI), tiriricão (Cyperus esculentus - CYPES) e capimcolchão (Digitaria horizontalis - DIGHO).
Na Figura 1 é mostrada a densidade das plantas daninhas no decorrer do período de avaliação dos tratamentos. Observa-se que os tratamentos que não receberam aplicação do herbicida apresentaram maiores médias de densidade de plantas daninhas. Já os tratamentos que receberam as doses do herbicida obtiveram médias de densidade de plantas daninhas reduzidas, podendo-se observar que, quanto maior a dose do herbicida, menor a densidade de plantas daninhas, independentemente da quantidade de palha aplicada.

Dessa forma, a palha de arroz sem herbicida não foi eficiente na redução da densidade de planta daninha, ao contrário dos resultados obtidos por Santos et al. (2001), em que a palha promoveu inibição na germinação de
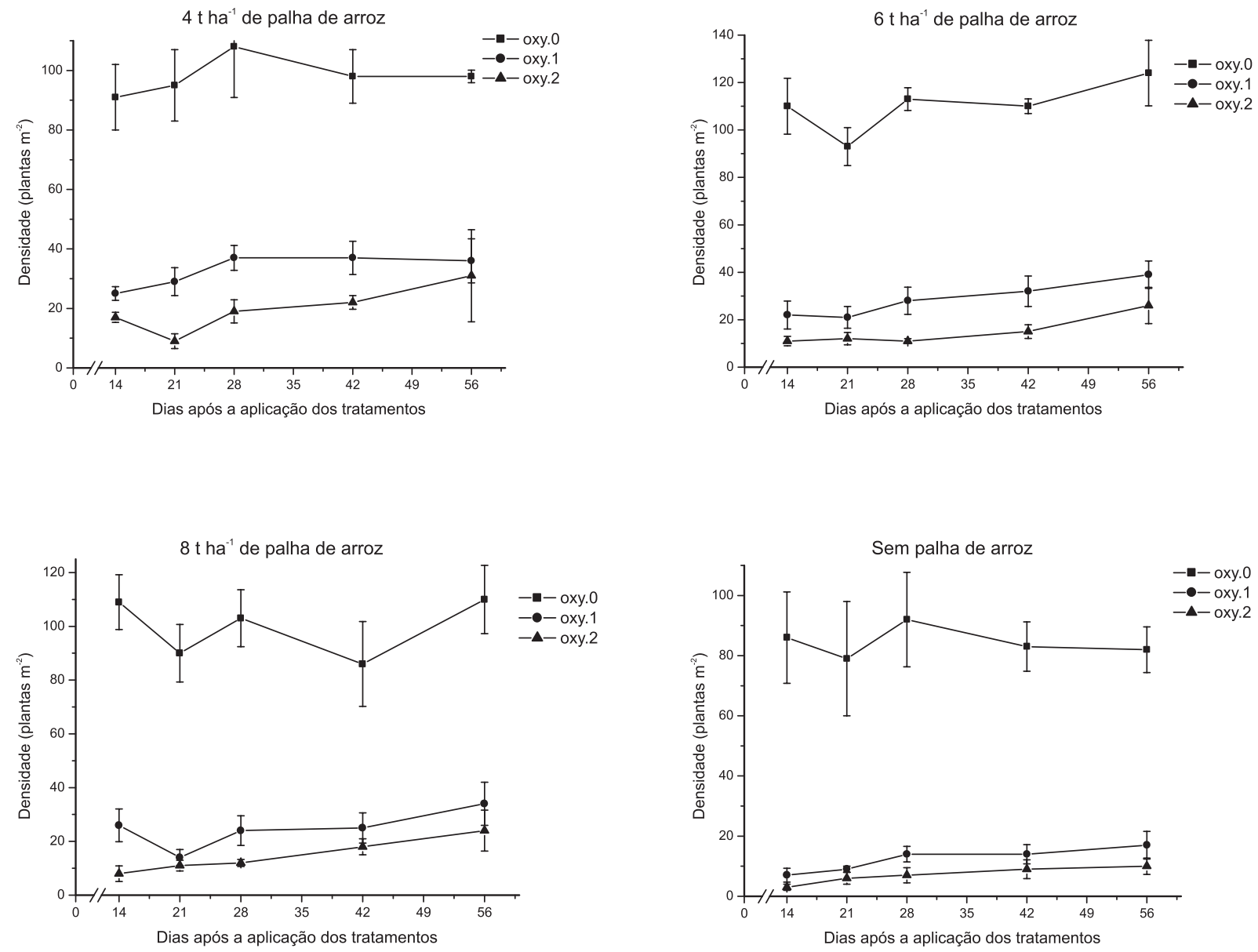

Figura 1 - Densidade de plantas daninhas em área experimental de sálvia (Salvia splendens) tratada com oxyfluorfen. Jaboticabal, 2007. 
sementes de caruru, e também por Araújo et al. (1993), que verificaram que a palha de arroz promoveu a maior redução no número de plantas daninhas no cultivo de alho.

Santos et al. (2001) comentam que a palha de arroz apresenta efeito alelopático e mostraram este efeito sobre a germinação de sementes de caruru, o que não foi evidenciado neste estudo; todavia, segundo Almeida (1991), a atividade alelopática depende diretamente da qualidade e quantidade do material vegetal depositado na superficie, do tipo de solo, da população microbiana, das condições climáticas e da composição específica da comunidade de plantas daninhas.

Já trabalhos realizados com palha de canade-açúcar têm mostrado resultados satisfatórios, como os realizados por Martins et al. (1999) e Correia \& Durigan (2004); no entanto, algumas espécies não foram afetadas, como Ipomoea quamoclit - Correia \& Durigan (2004) verificaram que a presença da palha incrementou a emergência das plântulas; e Cyperus rotundus - Durigan et al. (2004) observaram que a camada de palha correspondente a $10 \mathrm{t} \mathrm{ha}^{-1}$ reduziu a infestação, mas não evitou grandes densidades desta planta.

Como a infestação de plantas daninhas em canteiros nos jardins causa grande efeito visual de impacto negativo e como a utilização do herbicida veiculado à palha diminuiu os efeitos negativos deste, conclui-se que é viável a veiculação do herbicida oxyfluorfen à palha de arroz visando o controle de plantas daninhas em canteiros de sálvia. Todos os tratamentos com a veiculação do herbicida em palha apresentaram bons resultados, sem que efeitos fitotóxicos severos fossem observados nas plantas de sálvia. O tratamento que recebeu oxyfluorfen na dose de $2 \mathrm{~L} \mathrm{ha}^{-1}$ veiculado a $4 \mathrm{t} \mathrm{ha}^{-1}$ de palha de arroz apresentou resultados satisfatórios de controle de plantas daninhas e pequenos efeitos fitotóxicos em sálvia, permitindo sua recomendação. A pulverização direta do herbicida sobre o solo, embora apresente elevado controle das plantas daninhas, não se mostrou viável para a sálvia, principalmente até os 42 dias após a aplicação do produto. Após esse período, as plantas de sálvia recuperaram-se, apresentando-se vigorosas e úteis ao paisagismo.

\section{LITERATURA CITADA}

ALMEIDA, F. S. Controle de plantas daninhas em plantio direto. Londrina: IAPAR, 1991. 34 p. (Circular, 67).

ALVES, A. G. C.; COGO, N. P.; LEVIEN, R. Relações da erosão do solo com a persistência da cobertura vegetal morta. R. Bras. Ci. Solo, v. 19, n. 1, p. 127-132, 1995.

ALVES, L. W. R.; SILVA, J. B.; SOUZA, I. F. Efeito da aplicação de subdose dos herbicidas glyphosate e oxyfluorfen, simulando deriva sobre a cultura de milho (Zea mays L.) Ci. Agrotec., v. 24, n. 4, p. 889-897, 2000

ARAÚJO, R. C. et al. Efeitos da cobertura do solo sobre a cultura do alho (Allium sativum L.). Ci. Prática, v. 17, n. 3, p. 228-233, 1993

AZANIA, C. A. M. et al. Seletividade do imazapic para dois cultivares de amendoim (Arachis hypogaea) cultivados na ausência e na presença de palha de cana-de-açúcar. Planta Daninha, v. 22, n. 1, p. 145-150, 2004

AZANIA, C. A. M. et al. Desenvolvimento da tiririca (Cyperus rotundus) influenciado pela presença e ausência de palha de cana-de-açúcar e herbicida. Planta Daninha, v. 24, n. 1, p. 29-35, 2006.

BRAGAGNOLO, N.; MIELNICZUK, J. Cobertura do solo por palha de trigo e seu relacionamento com a temperatura e umidade do solo. R. Bras. Ci. Solo, v. 14, n. 3, p. 369-374, 1990.

CARTER, I.; JOHNSON, C. Influence of different types of mulches on eggplant production. HortScience, v. 23, n. 1, p. 143-145, 1988.

CORREIA, N. M.; DURIGAN, J. C. Emergência de plantas daninhas em solo coberto com palha de cana-de-açúcar. Planta Daninha, v. 22, n. 1, p. 11-17, 2004.

CREAGUR, R. A.; KATCHUR, D. An evaluation on plastic and fibrous materials as mulches for total control vegetation. HortScience, v. 10, n. 1, p. 482, 1975

DURIGAN, J. C. et al. Controle químico da tiririca (Cyperus rotundus) com e sem cobertura do solo pela palha de cana-de-açúcar. Planta Daninha, v. 22, n. 1, p. 127-135, 2004.

EUROPEAN WEED RESEARCH CONCIL - EWRC. Report of the $3^{\text {rd }}$ and $4^{\text {th }}$ meetings of EWRC - Committee of methods in weed research. Weed Res., v. 4, n. 1, p. 88, 1964. 
FIALHO, J. F.; BORGES, N. F.; BARROS, N. F. Cobertura vegetal e as características químicas e físicas e atividade da microbiótica de um Latossolo Vermelho-Amarelo distrófico. R. Bras. Ci. Solo, v. 15, n. 1, p. 21-28, 1991.

FREITAS, F. C. L. et al. Controle químico de brilhantina (Pilea microphylla) no cultivo de orquídeas. Planta Daninha, v. 25, n. 3, p. 589-593, 2007a.

FREITAS, F. C. L. et al. Controle de plantas daninhas na produção de mudas de plantas ornamentais. Planta Daninha, v. 25, n. 3, p. 595-601, 2007 b.

GRAVENA, R. et al. Controle de plantas daninhas através da palha de cana-de-açúcar associada à mistura dos herbicidas trifloxysulfuron sodium + ametrina. Planta Daninha, v. 22, n. 3, p. 419-427, 2004.

LORENZI, H.; SOUZA, H. M. Plantas ornamentais no Brasil - arbustivas, herbáceas e trapadeiras. Nova Odessa: Plantarum, 2001. 613 p.

MACIEL, C. D. G. et al. Seletividade de coroa-de-cristo (Euphorbia splendens) a diferentes classes de herbicidas para manejo de plantas daninhas em jardinagem. R. Bras. Hortic. Ornam., v. 11, n. 2, p. 132-140, 2005.

MARTINS, D. et al. Emergência em campo de dicotiledôneas infestantes em solo coberto com palha de canade-açúcar. Planta Daninha, v. 17, n. 1, p. 151-161, 1999.
MOREIRA, M. G. Utilização integrada de cobertura morta e pendimethalin ou oxyfluorfen no controle de plantas daninhas nas culturas da alface e da cebola. 1990. 55 f. (Trabalho apresentado como parte das exigências para graduação em Agronomia) - Universidade Estadual de São Paulo, Jaboticabal, 1990

PEREIRA, W. S. P. Herbicida de pré-emergência oxifluorfen. Série Técnica IPEF, v. 4, n. 12, p. 45-60, 1987.

RONCHI, C. P.; SILVA, A. A. Tolerância de mudas de café a herbicidas aplicados em pós-emergência. Planta Daninha, v. 21, n. 3 , p. $421-426,2003$.

SANTOS, J. C. F. et al. Influência alelopática das coberturas mortas de casca de café (Coffea arabica L.) e casca de arroz (Oryza sativa L.) sobre o controle do caruru-de-mancha (Amaranthus viridis L.) em lavoura de café. Ci. Agrotec., v. 25 , n. 5 , p. 1105-1118, 2001.

SEVERINO, F. J.; CHRISTOFFOLETI, P. J. Efeitos de quantidades de fitomassa de adubos verdes na supressão de plantas daninhas. Planta Daninha, v. 19, n. 1, p. 223-228, 2001.

YEN, J. H.; SHEU, W. S.; WANG, Y. S. Dissipation of the herbicide oxifluorfen in subtropical soils and its potential to contaminate groundwater. J. Ecotoxicol. Environ. Safety, v. 54, n. 2 , p. $151-156,2003$ 